Article

\title{
Association of Plasma Oligomerized Beta Amyloid with Neurocognitive Battery Using Korean Version of Consortium to Establish a Registry for Alzheimer's Disease in Health Screening Population
}

\author{
Jung-Ju Lee ${ }^{1}(0)$, Youngki Choi ${ }^{2}$, Soie Chung ${ }^{3}$, Dae Hyun Yoon ${ }^{4}$, Seung Ho Choi ${ }^{5}$, \\ Sung-Min Kang ${ }^{2}$, David Seo ${ }^{2}$ and Kyung-Il Park ${ }^{5,6, *}$ \\ 1 Department of Neurology, Nowon Eulji Medical Center, Eulji University, Seoul 01830, Korea; \\ sss331sss331@gmail.com \\ 2 Research and Development, PeopleBio Inc., Seongnam 13487, Korea; choi.youngki@peoplebio.net (Y.C.); \\ kang.sungmin@peoplebio.net (S.-M.K.); seo.david@peoplebio.net (D.S.) \\ 3 Department of Laboratory Medicine, Seoul National University Hospital Healthcare System Gangnam \\ Center, Seoul 06236, Korea; soiec@snuh.org \\ 4 Department of Psychiatrics, Seoul National University Hospital Healthcare System Gangnam Center, \\ Seoul 06236, Korea; dhyoon@snuh.org \\ 5 Healthcare Research Institute, Seoul National University Hospital Healthcare System Gangnam Center, \\ Seoul 06236, Korea; cshmed@snuh.org \\ 6 Department of Neurology, Seoul National University Hospital Healthcare System Gangnam Center, \\ Seoul 06236, Korea \\ * Correspondence: ideopki@gmail.com; Tel.: +82-2-2112-5756; Fax: +82-2-2112-5635
}

Received: 25 March 2020; Accepted: 17 April 2020; Published: 20 April 2020

\begin{abstract}
The increasing prevalence of Alzheimer's disease (AD) has become a global phenomenon presenting serious social and health challenges. For detecting early molecular changes in the disease, several techniques to measure varied species of amyloid beta in the peripheral blood have been recently developed, but the efforts to associate them with cognitive assessments have yet to produce sufficient data. We prospectively collected participants from the consecutive population who visited our center for brain health screening. In total, 97 participants $(\mathrm{F}: \mathrm{M}=58: 39)$ aged $69.4 \pm 7.52$ were assessed. Participants performed the Korean version of the Consortium to Establish a Registry for Alzheimer's disease (CERAD-K), the clinical dementia rating (CDR), plasma oligomeric amyloid- $\beta$ $(\mathrm{OA} \beta)$ level tests, routine blood tests, ApoE genotype, and brain MRI. Among total population, 55.7\% had a CDR of 0 , and $40.2 \%$ had a CDR of 0.5 . The results showed that word memory and word recall, and the total scores of the CERAD-K were negatively correlated with the plasma OA $\beta$ level. With a cut-off value of $0.78 \mathrm{ng} / \mathrm{mL}$ for the $\mathrm{OA} \beta$ level and a -1.5 standard deviation of age/sex/education adjusted norms for the CERAD-K; naming, word memory, word recall, word recognition, and total score were significantly correlated with the OA $\beta$ level. No correlation between the OA $\beta$ level and mini-mental status examination was found. Our results demonstrate that the level of plasma OA $\beta$ was well correlated with the measure of cognitive function through the CERAD-K in the field data collected from consecutive populations. Studies on longitudinal comparisons with large cohorts will further validate the diagnostic value of plasma $\mathrm{OA} \beta$ as a useful biomarker for screening $A D$ and predicting progression.
\end{abstract}

Keywords: Alzheimer's disease; blood biomarker; amyloid- $\beta$ protein; oligomer; cognitive assessment; CERAD 


\section{Introduction}

Alzheimer's disease (AD), one of the most common dementias, is a global health crisis. The number of people affected by the disease is projected to reach 13 million in the United States and 100 million globally by 2050 [1], with the prevalence rate increasing twofold every 20 years as a result of the aging population [2]. Symptoms of $\mathrm{AD}$, mainly characterized by cognitive impairment, start 18 years before a clinical diagnosis can be made [3]. As a result of its renowned prolonged preclinical stage [4], consistent efforts are being made to identify and diagnose AD earlier. The most well-established and validated biomarkers for $\mathrm{AD}$ are the cerebrospinal fluid (CSF) biomarker test and positron emission tomography (PET) [5]. These biomarkers, however, have limitations in terms of being utilized as universal $\mathrm{AD}$ diagnostics as they are invasive, expensive, and often unavailable in many healthcare systems. Consequently, the development of a blood-based biomarker, which can provide minimally invasive, simple, inexpensive test procedures, and high patient accessibility, has become a sensational breakthrough for researchers and industries worldwide. Extracellular accumulation of amyloid- $\beta$ $(\mathrm{A} \beta)$ and intracellular neurofibrillary tangles are the hallmarks of Alzheimer's disease. When amyloid precursor protein (APP) is cleaved by both gamma and beta secretase, an amino acid peptide called beta amyloid $(A \beta)$ is produced. $A \beta$ may abnormally fold into oligomeric forms, fibrillary forms and finally into plaques. Research into Alzheimer's disease has found that oligomerized $A \beta(O A \beta)$ are the most toxic among $A \beta$ species [6,7] and are highly associated with the pathogenesis of $A D[8,9]$. Various efforts are being made to detect oligomerized $A \beta$ in biofluid $[10,11]$ with the aim of validating it as the long-anticipated blood-based biomarker for AD diagnosis. Multimer Detection System-Oligomeric Amyloid- $\beta$ (MDS-OA $\beta$ ) [12] is a technique which measures the level of the $A \beta$ oligomerization $(A \beta O)$ tendency after spiking purified synthetic $A \beta$ into plasma samples. A previous study confirmed that the plasma OA $\beta$ level increases in plasma of $A D$ patients but does not increase in the plasma of healthy normal control [12]. The increase in the plasma $A \beta O$ level in AD highly correlates with CSF $A \beta 42$ and Pittsburgh compound B (PiB) PET standard uptake ratio [13]. In addition, the elevated plasma OA $\beta$ level showed a strong correlation with cognitive performance in patients with $\mathrm{AD}$, including an inverse correlation with scores on the mini-mental status examination (MMSE), cognitive abilities screening instrument, the common objects memory test, and a positive correlation with the Alzheimer's disease assessment scale-cognitive portion scores [14]. The level of these biomarkers has been reported to correlate with cognitive performance, especially in patients within the mild cognitive impairment (MCI)-AD continuum, but this association is still controversial and it is unclear whether this correlation is found even in non-AD dementia. The Consortium to Establish a Registry for Alzheimer's disease (CERAD), is a widely utilized neurocognitive assessment battery and is well validated in each country. Therefore, in this study, by comparing the MDS-OA $\beta$ value with the Korean version of Consortium to Establish a Registry for Alzheimer's disease CERAD-K, we aim to validate that the plasma OA $\beta$ level reflects the cognitive and memory function of individuals using the Korean version of CERAD (CERAD-K), and the potential for utilization of MDS-OA $\beta$ as a feasible diagnostic method to screen people in the prodromal stage or MCI stage.

\section{Materials and Methods}

\subsection{Study Population}

We prospectively and consecutively collected 100 participants who had planned to undergo a brain examination as part of the health checkup and agreed to perform additional blood sampling for MDS-OA $\beta$ in our center. The institutional review board of the Seoul National University Hospital approved this study, and a written informed consent was obtained.

\subsection{Plasma Oligomerized Beta Amyloid Assay}

In all participants, blood samples (about $8 \mathrm{~mL}$ ) were obtained in a heparin tube and immediately centrifuged at $1500 \times \mathrm{g}$ for $10 \mathrm{~min}$. Aliquots were stored at $-70{ }^{\circ} \mathrm{C}$ until they were analyzed with 
an inBlood ${ }^{\mathrm{TM}}$ oligomerized $\mathrm{A} \beta(\mathrm{OA} \beta)$ Test (Peoplebio Inc, Gyeonggi-do, Korea). This test utilizes a commercialized kit based on MDS-OA $\beta$ to quantify OA $\beta$ values. It is an atypical sandwich Enzyme-Linked Immunosorbent Assay (ELISA) using the epitope-overlapping antibodies specific for the $N$-terminus of beta amyloid $(\mathrm{A} \beta)$ to capture and detect plasma OA $\beta$. The epitopes for the $6 \mathrm{E} 10$ and W0-2-HRP antibodies overlapped at the N-terminus of $A \beta$, and mouse monoclonal anti-6E10 (BioLegend, San Diego, CA, USA) and anti-W0-2-HRP antibodies (Absolute Antibody Ltd., Oxford, $\mathrm{UK})$ were therefore used to capture and to detect $\mathrm{OA} \beta$, respectively.

Prior to the assay, aliquots of plasma samples were thawed at $37^{\circ} \mathrm{C}$ for $15 \mathrm{~min}$. All protocols were the same as in our previous papers $[12,15,16]$. As indicated in the assay protocol of the inBlood ${ }^{\mathrm{TM}}$ OAß Test, PBR-1 (purified synthetic A $\beta$ made by PeopleBio Inc.) was spiked into plasma and the mixture was incubated at $37^{\circ} \mathrm{C}$ for $48 \mathrm{~h}$. The incubated plasma sample mixture and serially diluted standard samples were added to each well of the plates. The plates were incubated at about 20 to $25^{\circ} \mathrm{C}$ for $1 \mathrm{~h}$. After washing three times with a washing buffer, the W02-HRP antibody was added to the wells, and the plates were incubated for $1 \mathrm{~h}$ at about 20 to $25^{\circ} \mathrm{C}$. To increase the sensitivity of detection, $100 \mu \mathrm{L} /$ well of enhanced chemiluminescence substrate solution (Rockland Immunochemicals Inc., Limerick, PA, USA) was added, and the Relative Luminescence Unit (RLU) signal was detected using a Victor $3^{\mathrm{TM}}$ multi-spectrophotometer. Dilutions providing signals in the linear range of the standard curves were used for the conversion to RLU values to determine the concentration of OA $\beta$. Cut-off values for MDS-OA $\beta$ were set as $0.78 \mathrm{ng} / \mathrm{mL}[15]$.

\subsection{Clinical Variables}

The presence of hypertension was defined by the fact that the subject was taking hypertensive medication. The presence of diabetes mellitus was assumed if the patient was taking diabetes medication or showed $\mathrm{HbA} 1 \mathrm{c} \geq 6.5 \%$ at the time of the MRI visit. Hyperlipidemia was defined as LDL-cholesterol $\geq 160 \mathrm{mg} / \mathrm{dl}$ or total-cholesterol $\geq 240 \mathrm{mg} / \mathrm{dl}$, or triglyceride $\geq 200 \mathrm{mg} / \mathrm{dl}$ at the time of visit. Information about smoking and alcohol drinking behavior was obtained based on routine questionnaires used at our center. Information about any history of hypertension, diabetes mellitus, and hyperlipidemia was also sought. At-risk drinking was defined according to The National Institute on Alcohol Abuse and Alcoholism (NIAAA) criteria [17]. Depression was screened through a Quick Inventory of Depressive Symptomatology-Self Report (QIDS-SR16), where equal or higher than 11 points indicated moderate to severe depression [18]. All subjects were interviewed and assessed clinically, including CDR and Global Deterioration Scale (GDS) tests by an experienced neurologist or psychiatrist. The CERAD-K test was performed on all subjects. The CERAD-K is composed of 8 subsets of cognitive function, which were verbal fluency, naming, mini-mental status examination (MMSE), word list memory, constructional praxis, word list recall, word list recognition, and praxis recall. The points from each subset were calculated according to its scoring system. Total II is a summed score excluding MMSE and Total I is a sum excluding both MMSE and the praxis recall score. For the dichotomized analysis, the abnormal CERAD-K in each subset and two types of total scores were defined as lower than -1.5 standard deviation of norms adjusting for age, sex, and education years. ApoE genotype testing was performed in 90 subjects. We also reviewed participants' brain MRI and MRA including detailed hippocampal sequence.

\subsection{Statistics}

Student's $t$-test was used for continuous variables, and the Mann-Whitney test was used unless the variables were normally distributed. Chi-square was used for di- or tri-chotomized categorical variables. Significance was set as $p<0.05$.

\section{Results}

Among 100 participants, we excluded three persons who had MRI lesions potentially affecting the neuropsychological test outcome. Their lesions were traumatic cerebromalacia on the right 
frontotemporal area, anterior cerebral infarction on the left hemisphere, and sizable cerebellar infarction, respectively.

\subsection{Aging Increases the Level of Oligomerized Beta Amyloid in Blood}

Overall, 97 subjects ( $\mathrm{F}: \mathrm{M}=58: 39)$ were analyzed and their age was $69.4 \pm 7.52$. The plasma oligomerized $\mathrm{A} \beta(\mathrm{OA} \beta)$ level was $0.70 \pm 0.24 \mathrm{ng} / \mathrm{mL}$. The average education period was 13.56 years. Apoe 4 carriers were 25 out of 90 persons $(27.8 \%)$. The plasma OA $\beta$ level was strongly correlated with aging in our consecutive population $(r=0.33, p=0.001)$ (Figure 1a). The number of subjects with clinical dementia rating (CDR) of 0 was $54(55.7 \%)$, with a CDR of 0.5 was $39(40.2 \%)$, with a CDR of 1 was two $(2.1 \%)$ and a CDR of 2 was two $(2.1 \%)$. The participants with a CDR of 0.5 met the criteria of $\mathrm{MCI}$ and the participants with a CDR of 0 were either cognitively healthy individuals or subjective cognitive decline (SCD) individuals. Taking into account that these participants were collected on the basis that they came to receive the brain examination, we found it rational to regard the whole group of participants with a CDR of 0 as SCD individuals. All subjects with a CDR of 0.5 showed 0.5 for the memory component score except one who obtained 1 for the memory score. Comparing groups between CDR0 and CDR0.5, the distribution of plasma OA $\beta$ level is not statistically different (Figure 1b).
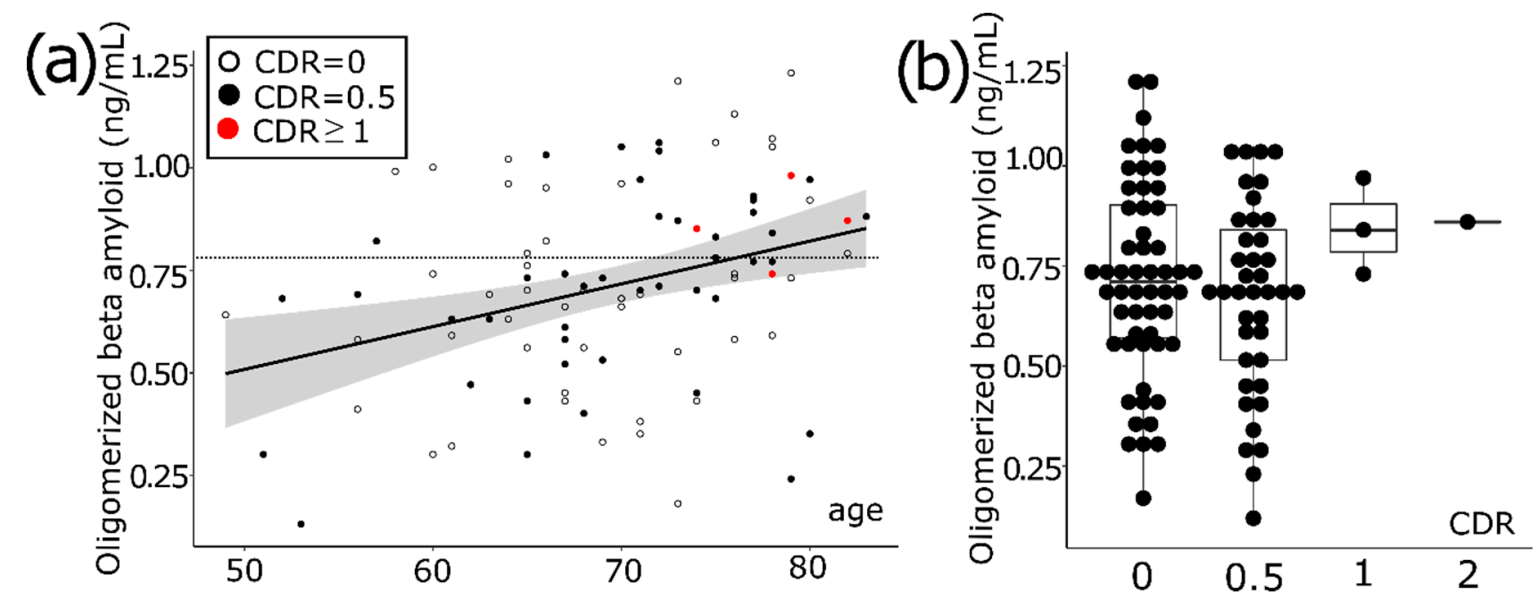

Figure 1. The distribution of plasma concentration of oligomerized beta amyloid (OA $\beta)$. (a) OA $\beta$ concentration increases with aging. The dotted line indicates a cut-off value of 0.78 . CDR indicates the clinical dementia rating. (b) Most participants have a clinical dementia rating (CDR) of 0 or a CDR of 0.5 . OA $\beta$ concentration was similar both in the CDR 0 and CDR 0.5 group.

\subsection{Comparison of High vs. Low $O A \beta$}

Using a cutoff of $0.78 \mathrm{ng} / \mathrm{mL}$ [15], 63 subjects were categorized into the high plasma OA $\beta$ level group and 34 were categorized into the low plasma OA $\beta$ level group. Age, sex, education period, the presence of Apoe4 haplotype, hypertension, diabetes, smoking, at-risk drinking, and QIDS-SR16 were not statistically different between the two groups (Table 1). There are more people with high GDS scores in the high OA $\beta$ group $(p=0.03)$.

Table 1. Demographics and clinical data of the population of high and low group of plasma OA $\beta$ levels.

\begin{tabular}{cccc}
\hline & High OA $\beta$ & Low OA $\beta$ & $p$ Value \\
\hline Age & $72.62 \pm 7.00$ & $67.67 \pm 7.27$ & 0.02 \\
\hline Sex (Female \%) & $20 / 34(58.8)$ & $38 / 63(60.3)$ & 0.89 \\
\hline Education (yrs) & $14.06 \pm 3.26$ & $13.30 \pm 4.05$ & 0.34 \\
\hline
\end{tabular}


Table 1. Cont.

\begin{tabular}{|c|c|c|c|}
\hline & High $O A \beta$ & Low $O A \beta$ & $p$ Value \\
\hline CDR & & & 0.24 \\
\hline $\mathrm{CDR}=0$ & $19 / 34$ & $35 / 63$ & \\
\hline $\mathrm{CDR}=0.5$ & $12 / 34$ & $27 / 63$ & \\
\hline $\mathrm{CDR} \geq 1$ & $3 / 34$ & $1 / 63$ & \\
\hline GDS & & & 0.03 \\
\hline GDS $=1$ & $18 / 34$ & $34 / 63$ & \\
\hline GDS $=2$ & $10 / 34$ & $28 / 63$ & \\
\hline GDS $\geq 3$ & $6 / 34$ & $1 / 63$ & \\
\hline Apoe4 carrier (\%) & $10 / 32$ & $15 / 58$ & 0.59 \\
\hline Hypertension & $12 / 34$ & $29 / 63$ & 0.26 \\
\hline Diabetes & $6 / 34$ & $12 / 63$ & 0.39 \\
\hline Hyperlipidemia & $21 / 34$ & $40 / 63$ & 0.39 \\
\hline Smoking & & & 0.25 \\
\hline Never & $25 / 34$ & $46 / 63$ & \\
\hline Ex & $8 / 34$ & $13 / 63$ & \\
\hline Current & $0 / 34$ & $4 / 63$ & \\
\hline At-risk drinking & $2 / 25$ & $13 / 58$ & 0.21 \\
\hline QIDS-SR(cutoff $\geq 11$ ) & $2 / 31$ & $4 / 57$ & 0.09 \\
\hline
\end{tabular}

3.3. OA Is Well Correlated with the Consortium to Establish a Registry for Alzheimer's Disease (CERAD-K) and its Subdomains

The plasma OA $\beta$ level was negatively correlated with raw scores of words list memory $(r=-0.29$. $p=0.003)$, word list recall $(r=-0.26, p=0.008)$, total I $(r=-0.24, p=0.02)$, and total II $(r=-0.23, p=0.02)$ (Figure 2a). We compiled five groups based on age/sex/education adjusting norms: 1) 1st group: $\geq$ mean score; 2) 2nd group: mean -1SD; 3) 3rd group: -1SD -1.5SD; 4) 4th group: -1.5 -2SD; 5) 5th group: $<-2.5 \mathrm{SD}$. According to these categories, the domains which were significantly correlated with the plasma OA $\beta$ level were naming $(r=0.23, p=0.02)$, word list memory $(r=0.32, p=0.002)$, word list recognition $(r=0.24, p=0.02)$, word list recognition $(r=0.23, p=0.03)$, total I $(r=0.25$, $p=0.01)$, and total II $(r=0.20, p=0.047)$ (Figure 2b). The best correlated domain was the 'word list memory'. This correlation was also evident when analyzing it in terms of dichotomized variables. We define "abnormal" as below $-1.5 \mathrm{SD}$ of sex/age/education adjusting norms in subtests as well as total score; the abnormality in four subtests, such as naming, word list memory/recall/recognition, and two types of total scores, was greater in the abnormal plasma OA $\beta$ group $(p=0.001,0.005,0.009$, $0.03,0.002$, and 0.01 ) (Figure 2c). However, any methods of analysis regarding MMSE score did not demonstrate a significant correlation with plasma OA $\beta$ level $(p=0.14$ for raw score, $p=0.36$ for age/sex/education-adjusted interval and $p=0.25$ for dichotomized analysis).

When we further analyzed the population with in the CDR 0 group, which is regarded as a cognitively healthy group, and the CDR 0.5 group, which is amnestic MCI separately, clinical variables such as age, the presence of hypertension, diabetes, hyperlipidemia, at-risk drinking, smoking, and education status were not statistically different between the groups (Table 2). 


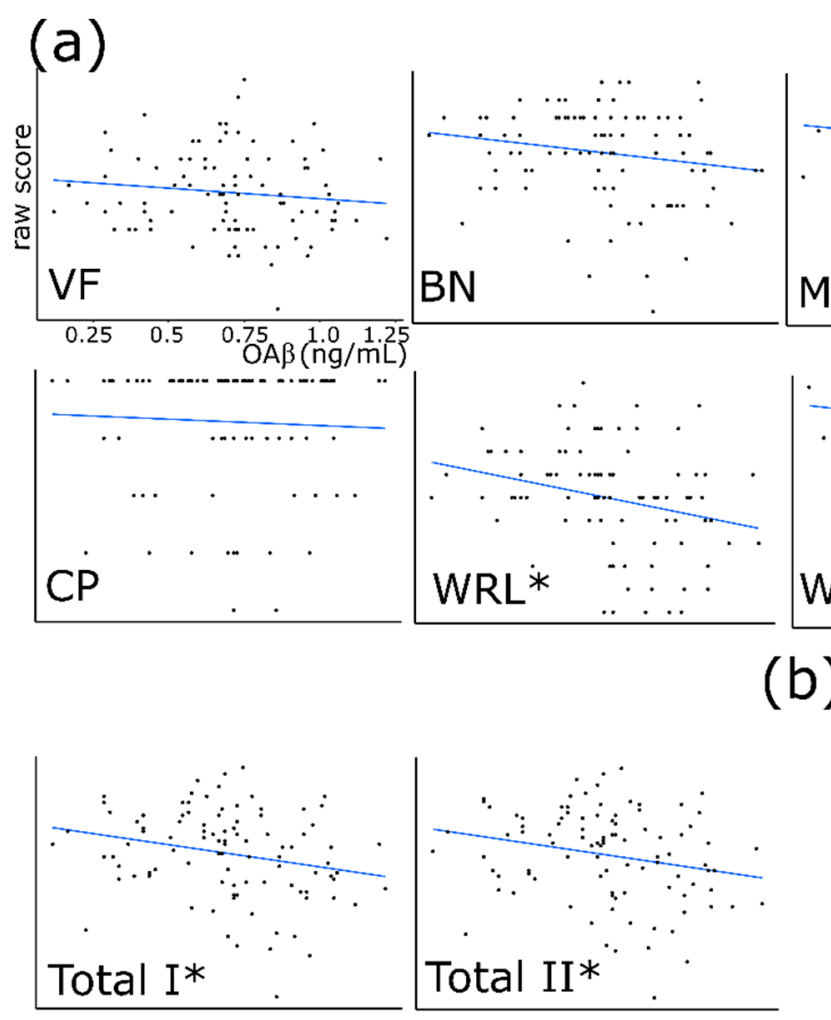

(c)

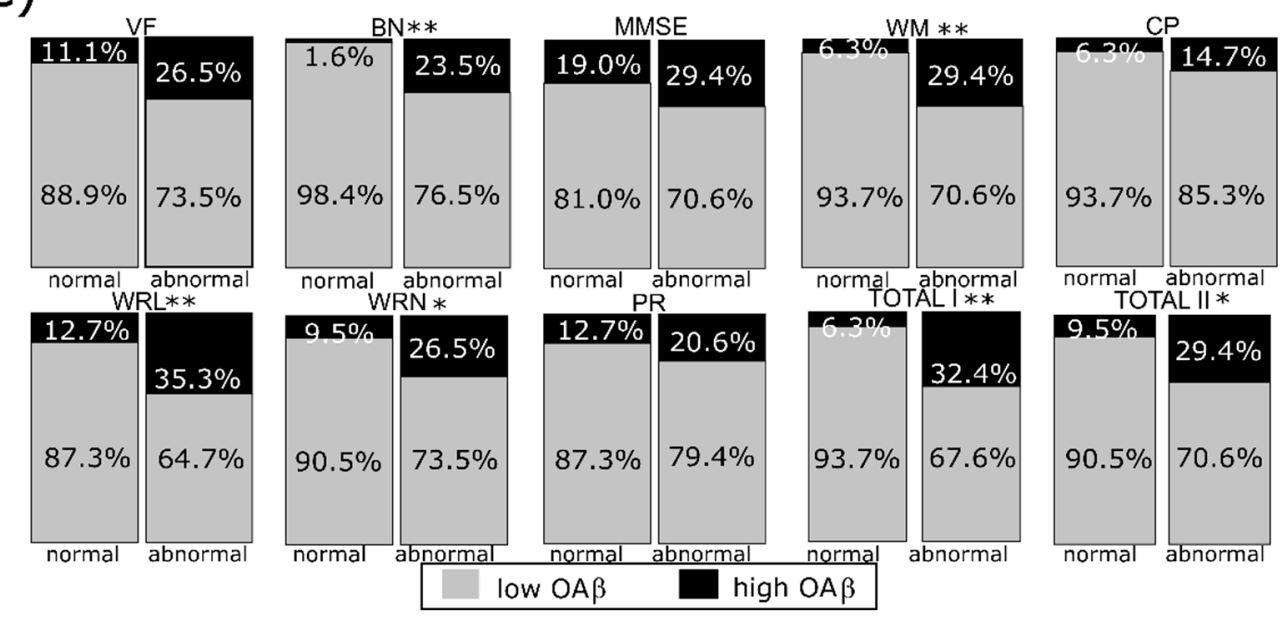

Figure 2. The subtests of the Korean version of Consortium to Establish a Registry for Alzheimer's disease (CERAD-K) and plasma oligomerized beta amyloid (OA $\beta$ ). (a) Raw scores of the CERAD-K and OA $\beta$ concentration. (b) The abnormal CERAD group (below -1.5 standard deviation of age/sex/education adjusting norms) in verbal fluency, naming, word memory/recall, and total scores showed higher OA $\beta$ concentration compared with control. (c) Abnormality in naming, word memory/recall/recognition, and total scores are significantly more frequent in high OA $\beta$ groups $(\geq 0.78 \mathrm{ng} / \mathrm{mL}$ ). SD—standard deviation; VF—verbal fluency; BN—Boston naming; MMSE-mini-mental status examination; WM-word list memory; $\mathrm{CP}$ - constructional praxis; WRL—word list recall; WRN-word list recognition; and PR-praxis recall. Total I is a sum of subtests except MMSE and PR and Total II is a sum except MMSE. ${ }^{*} p<0.05 .{ }^{* *} p<0.01$. 
Table 2. Clinical variables of population with $\mathrm{CDR}=0$ and $\mathrm{CDR}=0.5$.

\begin{tabular}{ccccc}
\hline & \multicolumn{2}{c}{ CDR $=\mathbf{0}(n=54)$} & \multicolumn{2}{c}{ CDR $=\mathbf{0 . 5}(n=39.95 \pm 7.90$} \\
\hline Age & \multicolumn{2}{c}{$69.07 \pm 7.14$} & High OA $\beta(n)$ & $p$ value \\
\hline Hypertension & $6 / 25$ & 0 value & $5 / 14$ & 0.72 \\
\hline Diabetes & $4 / 12$ & 1.00 & $2 / 6$ & 1.00 \\
\hline Hyperlipidemia & $11 / 35$ & 0.43 & $9 / 25$ & 0.48 \\
\hline Problem drinking & $2 / 9$ & 0.65 & $0 / 6$ & 0.30 \\
\hline Smoking & & 0.74 & 0.46 \\
\hline None & $14 / 40$ & & $10 / 29$ & \\
\hline Ex & $5 / 13$ & & $2 / 7$ & \\
\hline Current & $0 / 1$ & $0 / 3$ & 0.69 \\
\hline $\begin{array}{c}\text { QIDS-SR } \\
(\geq \text { moderate })\end{array}$ & $2 / 7$ & 0.62 & $2 / 9$ & 1.0 \\
\hline Apoe4 carrier & $6 / 15$ & 0.70 & $2 / 8$ \\
\hline Education $(<12$ yrs $)$ & $4 / 10$ & 0.73 & $2 / 8$ \\
\hline
\end{tabular}

\section{Discussion}

The cerebrospinal fluid (CSF) biomarker test and PiB PET are excellent in terms of diagnostic accuracy, but there are limitations for these biomarkers in terms of being widely utilized in early diagnosis of $\mathrm{AD}$ due to the high invasiveness, high risk of side effects, and restricted availability to patients. Over the years, much effort has been devoted to global research and as a result several blood-based biomarkers have been developed $[19,20]$. These blood-based biomarkers have demonstrated high correlation between the level of $\mathrm{A} \beta 42, \mathrm{~A} \beta 40$, and the risk of $\mathrm{AD}[21,22]$, but they require highly sensitive equipment to detect low-concentrated target proteins. On the other hand, MDS-OA $\beta$ utilizes spiking of purified synthetic $A \beta$ into plasma and incubation thereafter to measure the level of $A \beta O$ tendencies in plasma [12]. The comparative measurement on the level of $A \beta O$ tendencies in $\mathrm{AD}$ and normal plasma using MDS-OA $\beta$ was conducted and the resulting values were $1.43 \pm 0.30 \mathrm{ng} \mathrm{ml}$ and $0.45 \pm 0.19 \mathrm{ng} / \mathrm{mL}$, respectively, showing the significant difference between the two groups $(p<0.001)$ [15]. Reference interval was induced and the cut-off value was validated according to the guideline of Clinical Laboratory Standard Institute C28-A3, the 95\% reference interval applied to the normal group was $0.783 \mathrm{ng} / \mathrm{mL}$ and it was very close to the cut-off value of receiver operating characteristic (ROC) curve analysis. Applying the cut-off of $0.78 \mathrm{ng} / \mathrm{mL}$, area under curve (AUC) was 0.999 , sensitivity was $100 \%$ and specificity was $92.3 \%$, showing high accuracy in terms of AD diagnosis [15]. In this study, the MDS-OA $\beta$ value positively correlated with age, contrary to the results showing no correlation with age in community-based normal controls [15]. This difference might be due to the fact that most people who came to the healthcare center and participated in this study voluntarily were potentially SCD population. This also seems to affect the result that the level of $\mathrm{OA} \beta$ is not significantly different both in the groups of $\mathrm{CDR}=0$ and $\mathrm{CDR}=0.5$.

The quality of this study lies in the fact that it validated the relationship between MDS-OA $\beta$ and the CERAD-K, a Korean version of the standardized neuropsychological evaluation and diagnosis of AD [23]. Rossetti et al. [24] described the CERAD total score in AD patients, and the normal control for four years showed a significantly greater decrease in AD patients than in the normal group. Hwang et al. [25] confirmed that the CERAD scores decreased as the disease progressed through three years of follow-up of participants in the cognitive normal, SCD, MCI, and ADD groups, and CSF A $\beta 42$ levels in each group also decreased in correlation. In 2019, Meng et al. [14] described that an increase in MDS-OA $\beta$ levels in AD patients correlated with episodic memory loss. Other studies have reported that scores on language-related evaluative tasks such as word list memory and word list recall reflect AD-associated cognitive and memory function changes $[25,26]$. Furthermore, the decrease 
in the CERAD score in the order of normal individuals, MCI, and AD correlated with the atrophy of white matter lesions and gray matter in the brain [27], and scores for the word list recall and memory from the CERAD had a close relationship with atrophy in the medial temporal lobe [28]. In addition, the study by Youn et al. [16] confirmed that the correlation between the change in the MDS-OA $\beta$ value and brain atrophy in the form of $\mathrm{AD}$ exists. The cross-sectional and longitudinal evidence of connections among neurodegeneration, blood $\mathrm{OA} \beta$, and cognitive impairment in predementia status should be sought in future studies.

The significance of this study lies in the fact that the results are based on field data collected from the consecutive population. However, the main limitation is the small number of participants in the study. We understand the necessity of future studies using more refined global cognitive measures, larger samples with non-AD dementias, and ultimately a longitudinal design to establish the mechanisms for cognitive impairment caused by AD. The longitudinal studies of test-based cognitive performance assessments, along with blood biomarkers for people at high risk of dementia, are crucial to understanding cognitive decline mechanisms and improving AD diagnostic accuracy in the future.

Author Contributions: Conceptualization, J.-J.L., D.H.Y., S.H.C., S.-M.K., and K.-I.P.; Methodology, Y.C., S.C., S.-M.K., D.S., and K.-I.P.; Validation, D.H.Y., S.H.C., and K.-I.P.; Formal Analysis, S.C., Y.C., D.S., and K.-I.P.; Investigation, K.-I.P.; Resources, D.H.Y., S.-M.K., and K.-I.P.; Data Curation, Y.C., D.H.Y., and K.-I.P; Writing-Original Draft Preparation, J.-J.L., Y.C., D.S., and K.-I.P.; Writing-Review \& Editing, J.-J.L., Y.C., S.C., D.H.Y., S.H.C., and K.-I.P.; Visualization, All authors; Supervision, K.-I.P.; Project Administration, K.-I.P.; Funding Acquisition, K.-I.P. All authors have read and agreed to the published version of the manuscript.

Funding: This research was funded by Peoplebio inc. (K.-I.P.).

Conflicts of Interest: The sponsors had no role in the design, execution, or interpretation. The authors (Y.C. \& D.S.) only wrote a technical part of the laboratory analysis.

\section{References}

1. Cummings, J.L.; Lee, G.; Ritter, A.; Sabbagh, M.; Zhong, K. Alzheimer's disease drug development pipeline: 2019. Alzheimers Dement. 2019, 5, 272-293. [CrossRef]

2. Alzheimer's Association. 2015 Alzheimer's disease facts and figures. Alzheimers Dement. 2015, 11, 332-384. [CrossRef] [PubMed]

3. Rajan, K.B.; Wilson, R.S.; Weuve, J.; Barnes, L.L.; Evans, D.A. Cognitive impairment 18 years before clinical diagnosis of Alzheimer disease dementia. Neurology 2015, 85, 898-904. [CrossRef] [PubMed]

4. Jack, C.R., Jr.; Albert, M.S.; Knopman, D.S.; McKhann, G.M.; Sperling, R.A.; Carrillo, M.C.; Thies, B.; Phelps, C.H. Introduction to the recommendations from the National Institute on Aging-Alzheimer's Association workgroups on diagnostic guidelines for Alzheimer's disease. Alzheimers Dement. 2011, 7, 257-262. [CrossRef] [PubMed]

5. Jack, C.R., Jr.; Bennett, D.A.; Blennow, K.; Carrillo, M.C.; Dunn, B.; Haeberlein, S.B.; Holtzman, D.M.; Jagust, W.; Jessen, F.; Karlawish, J.; et al. NIA-AA Research Framework: Toward a biological definition of Alzheimer's disease. Alzheimers Dement. 2018, 14, 535-562. [CrossRef]

6. Um, J.W.; Nygaard, H.B.; Heiss, J.K.; Kostylev, M.; Stagi, M.; Vortmeyer, A.; Wisniewski, T.; Gunther, E.C.; Strittmatter, S.M. Alzheimer amyloid- $\beta$ oligomer bound to postsynaptic prion protein activates Fyn to impair neurons. Nat. Neurosci. 2012, 15, 1227-1235. [CrossRef]

7. Cline, E.N.; Bicca, M.A.; Viola, K.L.; Klein, W.L. The Amyloid- $\beta$ Oligomer Hypothesis: Beginning of the Third Decade. J. Alzheimers Dis. 2018, 64, S567-S610. [CrossRef]

8. Koffie, R.M.; Meyer-Luehmann, M.; Hashimoto, T.; Adams, K.W.; Mielke, M.L.; Garcia-Alloza, M.; Micheva, K.D.; Smith, S.J.; Kim, M.L.; Lee, V.M.; et al. Oligomeric amyloid beta associates with postsynaptic densities and correlates with excitatory synapse loss near senile plaques. Proc. Natl. Acad. Sci. USA 2009, 106, 4012-4017. [CrossRef]

9. Haass, C.; Selkoe, D.J. Soluble protein oligomers in neurodegeneration: Lessons from the Alzheimer's amyloid $\beta$-peptide. Nat. Rev. Mol. Cell Boil. 2007, 8, 101-112. [CrossRef]

10. Fukumoto, H.; Tokuda, T.; Kasai, T.; Ishigami, N.; Hidaka, H.; Kondo, M.; Allsop, D.; Nakagawa, M. High-molecular-weight beta-amyloid oligomers are elevated in cerebrospinal fluid of Alzheimer patients. FASEB J. 2010, 24, 2716-2726. [CrossRef] 
11. Sun, L.; Zhong, Y.; Gui, J.; Wang, X.; Zhuang, X.; Weng, J. A hydrogel biosensor for high selective and sensitive detection of amyloid-beta oligomers. Int. J. Nanomed. 2018, 13, 843-856. [CrossRef] [PubMed]

12. An, S.S.A.; Lee, B.S.; Yu, J.S.; Lim, K.; Kim, G.J.; Lee, R.; Kim, S.; Kang, S.; Park, Y.H.; Wang, M.J.; et al. Dynamic changes of oligomeric amyloid beta levels in plasma induced by spiked synthetic $\mathrm{A} \beta_{42}$. Alzheimers Res. Ther. 2017, 9, 86. [CrossRef] [PubMed]

13. Wang, M.J.; Yi, S.H.; Han, J.; Park, S.Y.; Jang, J.-W.; Chun, I.K.; Kim, S.E.; Lee, B.S.; Kim, G.J.; Yu, J.S.; et al. Oligomeric forms of amyloid-beta protein in plasma as a potential blood-based biomarker for Alzheimer's disease. Alzheimers Res. Ther. 2017, 9, 98. [CrossRef] [PubMed]

14. Meng, X.; Li, T.; Wang, X.; Lv, X.; Sun, Z.; Zhang, J.; Yu, X. Association between increased levels of amyloid-beta oligomers in plasma and episodic memory loss in Alzheimer's disease. Alzheimers Res. Ther. 2019, 11, 89. [CrossRef] [PubMed]

15. Kim, S.; Youn, Y.C.; Lee, B.S.; Kim, G.J.; Ryu, J.S.; Lim, K.; Ryu, N. Blood amyloid- $\beta$ oligomerization as a biomarker of Alzheimer's disease: A blinded validation study. J. Alzheimers Dis. 2020, in press.

16. Youn, Y.C.; Kang, S.; Suh, J.; Park, Y.H.; Kang, M.J.; Pyun, J.M.; An, S.S.A. Blood amyloid-beta oligomerization associated with neurodegeneration of Alzheimer's disease. Alzheimers Res. Ther. 2019, 11, 40. [CrossRef]

17. National Institute on Alcohol Abuse and Alcoholism (NIAAA). Drinking Levels Defined. 2016. Available online: https://www.niaaa.nih.gov/alcohol-health/overview-alcohol-consumption/moderate-binge-drinking (accessed on 25 March 2020).

18. Rush, A.J.; Trivedi, M.H.; Ibrahim, H.M.; Carmody, T.J.; Arnow, B.; Klein, D.N.; Markowitz, J.C.; Ninan, P.T.; Kornstein, S.; Manber, R.; et al. The 16-Item Quick Inventory of Depressive Symptomatology (QIDS), clinician rating (QIDSC), and self-report (QIDS-SR): A psychometric evaluation in patients with chronic major depression. Biol. Psychiatry 2003, 54, 573e83. [CrossRef]

19. Janelidze, S.; Stomrud, E.; Palmqvist, S.; Zetterberg, H.; Van Westen, D.; Jeromin, A.; Blennow, K. Plasma $\beta$-amyloid in Alzheimer's disease and vascular disease. Sci. Rep. 2016, 6, 1-11. [CrossRef]

20. Nakamura, A.; Kaneko, N.; Villemagne, V.L.; Kato, T.; Doecke, J.; Doré, V.; Tomita, T. High performance plasma amyloid- $\beta$ biomarkers for Alzheimer's disease. Nature 2018, 554, 249. [CrossRef]

21. Lue, L.F.; Guerra, A.; Walker, D.G. Amyloid Beta and Tau as Alzheimer's Disease Blood Biomarkers: Promise From New Technologies. Neurol. Ther. 2017, 6 (Suppl. 1), 25-36. [CrossRef]

22. Zhou, L.; Chan, K.H.; Chu, L.W.; Kwan, J.S.; Song, Y.Q.; Chen, L.H.; Ho, P.W.; Cheng, O.Y.; Ho, J.W.; Lam, K.S. Plasma amyloid-beta oligomers level is a biomarker for Alzheimer's disease diagnosis. Biochem. Biophys. Res. Commun. 2012, 423, 697-702. [CrossRef]

23. Moms, J.C.; Heyman, A.; Mohs, R.C.; Hughes, J.P.; van Belle, G.; Fillenbaum, G.; Mellits, E.D.; Clark, C. The Consortium to Establish a Registry for Alzheimer's Disease (CERAD). Part I. Clinical and neuropsychological assessment of Alzheimer's disease. Neurology 1989, 39, 1159-1165. [CrossRef] [PubMed]

24. Rossetti, H.C.; Cullum, C.M.; Hynan, L.S.; Lacritz, L. The CERAD Neuropsychologic Battery Total Score and the progression of Alzheimer disease. Alzheimer Dis. Assoc. Disord. 2010, 24, 138-142. [CrossRef] [PubMed]

25. Hwang, J.; Jeong, J.H.; Yoon, S.J.; Park, K.W.; Kim, E.J.; Yoon, B.; Jang, J.W.; Kim, H.J.; Hong, J.Y.; Lee, J.M.; et al. Clinical and Biomarker Characteristics According to Clinical Spectrum of Alzheimer's Disease (AD) in the Validation Cohort of Korean Brain Aging Study for the Early Diagnosis and Prediction of AD. JCM 2019, 8, 341. [CrossRef] [PubMed]

26. Karrasch, M.; Sinervä, E.; Grönholm, P.; Rinne, J.; Laine, M. CERAD test performances in amnestic mild cognitive impairment and Alzheimer's disease. Acta Neurol. Scand. 2005, 111, 172-179. [CrossRef]

27. Bilello, M.; Doshi, J.; Nabavizadeh, S.A.; Toledo, J.B.; Erus, G.; Xie, S.X.; Trojanowski, J.Q.; Han, X.; Davatzikos, C. Correlating Cognitive Decline with White Matter Lesion and Brain Atrophy Magnetic Resonance Imaging Measurements in Alzheimer's Disease. J. Alzheimers Dis. 2015, 48, 987-994. [CrossRef]

28. Kwon, J.W.; Kim, H.; Lee, K.J. Association between Cognitive Function, Behavioral and Psychological Symptoms of Dementia and White Matter Hyperintensities in Patients with Alzheimer's Disease and Mild Cognitive. KJPM 2018, 26, 2119.

(C) 2020 by the authors. Licensee MDPI, Basel, Switzerland. This article is an open access article distributed under the terms and conditions of the Creative Commons Attribution (CC BY) license (http://creativecommons.org/licenses/by/4.0/). 